\title{
The robot that can achieve card magic
}

\author{
Ryoma Koretake*, Makoto Kaneko and Mitsuru Higashimori
}

\begin{abstract}
We succeeded in developing a robot that can play a card magic for the first time in the world. The robot can complete a magic by quickly switching the first-card-dealing and the-second-card-dealing. This paper is composed of three parts where one is the design of the robot by referring human motion, the second is the mechanics of the card manipulation by utilizing a simplified model, and a magic actually done by the robot. We would note that as far as the card dealing is concerned, the developed robot is even faster than human magician.
\end{abstract}

Keywords: Robot magic; Card manipulation; Hyper magic

\section{Background}

There are various kinds of card magic, such as secondcard-dealing [1] where the second card is taken while the top card keeps stationary, bottom-card-dealing [2] where the bottom card is taken by pretending finger motion for taking the top card, and buckeye [3] where plural cards are simultaneously manipulated. Skillful card dealing by finger is a key for demonstrating a beautiful performance of card magic. Professional card magicians can achieve various basic motions, such as sliding, picking, and releasing card one by one under an appropriately force and position control by finger. In case of card magician, these skills can be acquired through practice again and again. As far as we have examined conventional literature, there have been no work discussing robotic card magician. We believe that the concept of robot magician itself opens a new business chance, especially in entertainment area. By playing magic cooperatively between human and robot magicians, we can make a new world of magic entertainment where the conventional magic show can not do.

Under these backgrounds, the goal of this paper is to propose a hyper magician robot that can achieve the card magic by quickly switching the second-card-dealing and the first-card-dealing. For designing and developing such a robot, what is the key? Extracting the essential functions from human motion during card magic may provide us with a good hint for designing such a robot. We would note that our basic stance is not to imitate the human motion but to extract the basic functions from human

\footnotetext{
*Correspondence: koretake@hh.mech.eng.osaka-u.ac.jp Department of Mechanical Engineering, Osaka University, 2-1 Yamadaoka, Suita, Osaka 565-0871, Japan
}

motion and implement them into the robot by completely cutting extra functions with second order priority.

Finally, we developed the hyper magician with two active degrees of freedom and one passive degree of freedom. Through experiments for the second-card-dealing by utilizing the developed hyper magician, we succeeded in achieving it with the speed of six cards/sec and in playing magic by quickly switching the first-card-dealing and the second-card-dealing (see the Additional file 1: video clip). This paper is organized as follows. After introduction, we briefly review conventional works where we explain a couple of key technologies which are closely related to hyper magician robot. Then, we analyze the finger motion during card manipulation of human for obtaining the deformed robot model and show the basic concept of robot design. Then, we discuss the mechanics of the second-card-dealing by using a simplified model. Next, we explain the experimental model and results precisely, before concluding remarks.

\section{Related works}

There have been many magics [4-6]. Card magic is one of them. As far as we know, there have been no works concerning with card magic by a robot, so far, while there are a couple of works $[7,8]$ in the HRI research field. On the other hand, there have been many fundamental works on robotic finger leading to card dealing, such as sliding motion based manipulation [9-12], pushing based manipulation [13-15], rolling motion manipulation $[16,17]$, and modeling of soft finger tip $[18,19]$. Especially, works on soft finger modeling provide us with a good hint from the viewpoint of supporting both frictional

\section{是 Springer}

(c) 2015 Koretake et al.; licensee Springer. This is an Open Access article distributed under the terms of the Creative Commons Attribution License (http://creativecommons.org/licenses/by/4.0), which permits unrestricted use, distribution, and reproduction in any medium, provided the original work is properly credited. 
force and moment, since this type of finger tip can resist frictional moment around the axis perpendicular to the contact surface as well as frictional force. While a number of multi-fingered robot hands [20-23] have been designed and developed so far, most of them move very slowly due to multiple coordination control under mechanical constraint condition. On the other hand, there have been a couple of works where they pursue quick response by reducing the active degrees of freedom as many as possible $[24,25]$. In order to speed up card manipulation, these robots also provide us with a good hint.

\section{Human finger motion during the second-card-dealing}

Figure 1 shows an example of a series of finger motion in the second-card-dealing by magician. From Figure 1, we can learn that the magician is grasping the bundle of cards by the fingers of left hand and taking a card one by one by using the fingers of right hand. One interesting observation from Figure 1 is that the thumb in the left hand has two functions where one is for grasping the bundle of card and the other is for slightly moving the top card just before the right hand pick up the second card, so that the second top card can be easily taken. Without this thumb motion, it is hard for the fingers of right hand to smoothly take the second card one by one. As for the robot application, this function can be realized by implementing one finger capable of slightly rotating the top card. For simplicity, suppose only three cards. Figure 2 shows a set of possible forces acting on the second card during the second-carddealing where red and black arrows denote the normal and

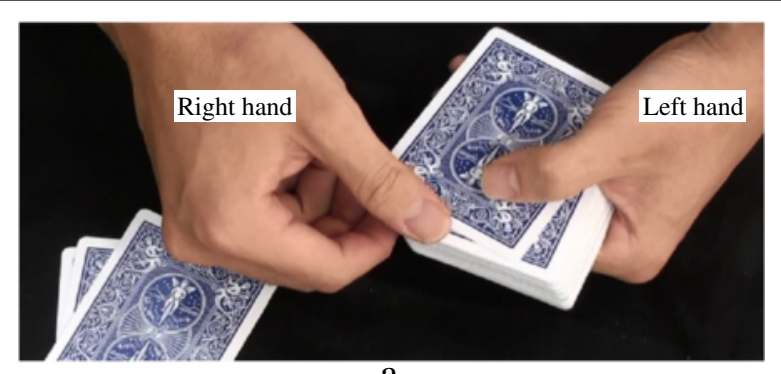

a

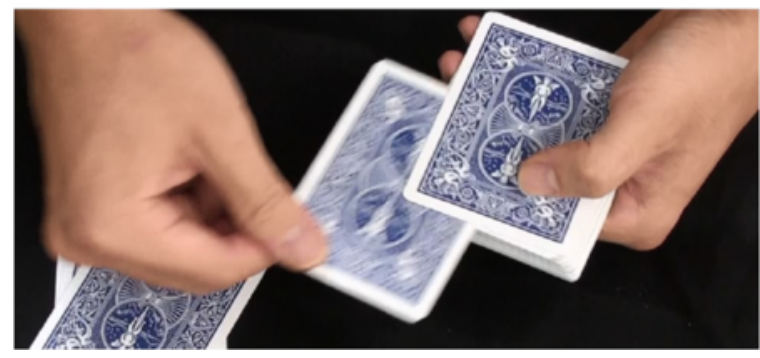

b

Figure 1 Second card dealing by magician: "Right" and "left" hand are from the magician viewpoint, respectively.

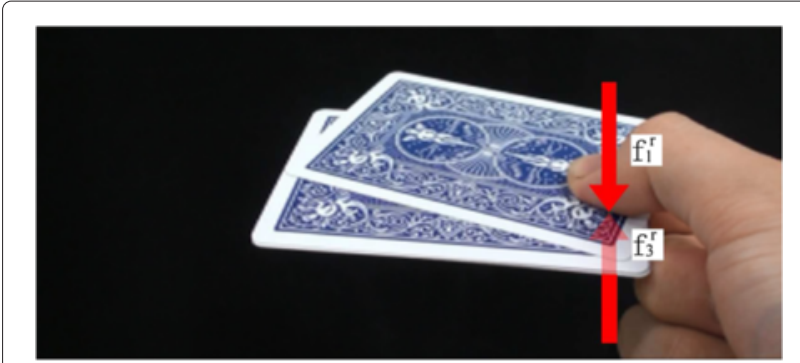

a

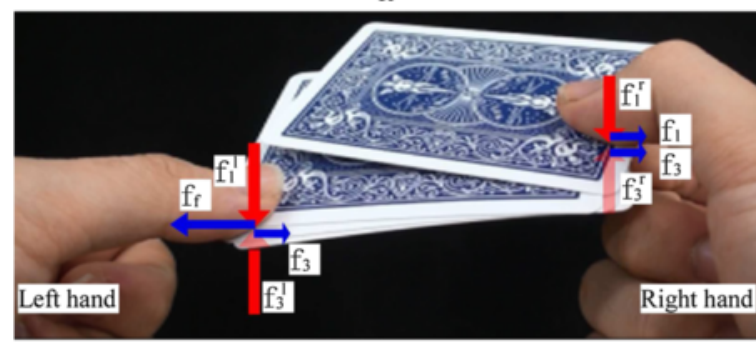

b

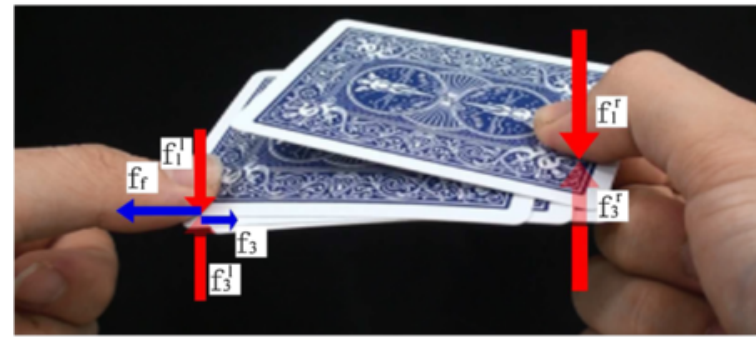

c

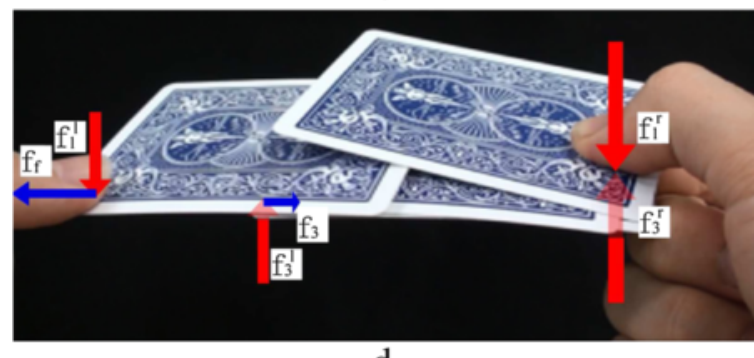

d

Figure 2 Forces during the second-card dealing: "Right" and "left" hand are from the magician viewpoint, respectively. Red and black arrows denote the normal and tangential forces, respectively, and the subscript " 1 ", " 3 ", and "f" are forces acted by the top card, the bottom card, and by the finger, respectively, and the superscript" $r$ ", and "I" are the right and the left finger, respectively.

tangential forces, respectively, and the subscript " 1 ", " 3 ", and "f" are forces acted by the top card, the bottom card, and by the finger, respectively, and the superscript "r", and "l" are the right and the left finger, respectively. Figure 2(a) shows that three cards are firmly grasped by two right fingers. Figure 2(b) shows that the second card is pulled by the left finger in Figure 2(b) while three cards are still grasped by two right fingers. We would note that once the second card starts to slide, the second card receives the friction forces by both the left finger and the bottom card. 
Figure 2(c) shows that the second card is released from the constraint area of two right fingers. Figure 2(d) shows that the second card is further moved by the left finger and the constraint by the right finger is completely released from two right fingers. As a result, the second card can be easily removed from the card bundle. Under the state in Figure 2(d), the second card mainly receives both normal and tangential forces from both the left finger and the third card, as shown in Figure 2(d).

\section{Basic concept of robot design}

Based on the discussions given in the earlier chapter, let us now discuss the robot design. Figure 3 shows an overview of the hyper magician robot, where Figure 3(a) and (b) show a hand model learnt by human and its highly deformed model, respectively. The deformed model includes four fingers, where Finger 1, 2, 3 and 4 correspond to the thumb of the left hand, the remaining four fingers of the left hand, the thumb of the right hand, and the index finger of the right hand in Figure 1, respectively. The deformed model is composed of two parts; one is for grasping a bundle of cards and the other one is for manipulating a card one by one from the bundle. Two parts are called "grasping part" and "manipulation part", respectively while the grasping part still includes an actuator for rotating the card through friction. The grasping part is composed of Finger 1 and Finger 2 where both Fingers are

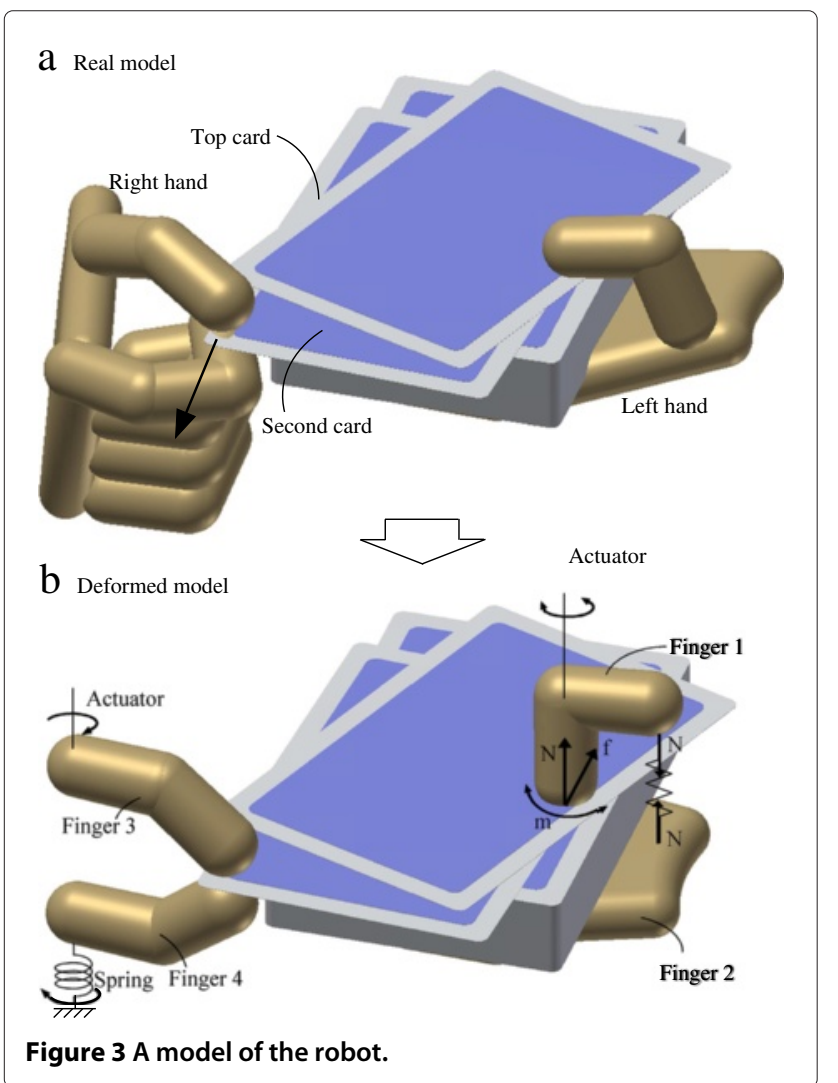

connected by a spring as shown in Figure 3(b), so that we can generate an appropriate internal force for grasping. The manipulation part is composed of Finger 3 and Finger 4 where Finger 3 includes an actuator, while Finger 4 has a spring without any actuator as shown in Figure 3(b). There are a couple of technical challenges where one is how to take the second card sandwiched by the top and the third card, and the second one is how to make the second card slide translationally by Finger 3 while other cards are kept stationary as much as possible and the third issue is how to eventually take the second card for completely moving out from the bundle. We discuss these issues one by one.

\section{Mechanics of the second-card-dealing}

Can we remove the second card without keeping both the top and the third cards stationary? This is an interesting question for discussing the second-card-dealing. If we can find the condition, it will provide us with a good guideline for designing the robot hardware, especially from the view point of the surface material. In this chapter, we try to find an answer by using a simplified model as shown in Figure 4 where $N, F$, and $\mu$ are the normal force, the pulling force, and frictional coefficient, respectively, and the subscript "s" and " $\mathrm{d}$ " are static and dynamic frictional coefficient, respectively. We assume that the frictional coefficient is equal between cards but different between card and the table, and between card and the finger being in contact with the top card. To simplify the discussion, we assume $\mu_{s}^{\prime}>\mu_{s}$ where $\mu_{s}^{\prime}$ denotes the static frictional coefficient between the top card and the finger being in contact with the top card. Additionally, we suppose $\mu_{s}>\mu_{d}$, which is naturally well accepted. Figure 4 shows two models where Figure 4(a) supposes that the second card is moving at the initial condition and Figure 4(b) supposes that all cards are stationary at the initial condition. To further simplify the discussion, we suppose $F=N$, which means that both normal force and the pulling force are equal each other. We would note that this condition can be actually realized. Under this condition, let us consider the case as shown in Figure 4(a). Since the second card is moving at the initial condition, we can continuously remove the second card under $\mu_{s}>\mu_{d}$, as shown in Figure 4(a). Now, let us first discuss the case as shown in Figure 4(b). The condition for removing the second card is given by $F>2 \mu_{s} F$, which means $\mu_{s}<0.5$. The condition for keeping the top card stationary is given by $\mu_{s}^{\prime} F>\mu_{s} F$. By assumption, $\mu_{s}^{\prime}>\mu_{s}$ always holds. As for the third card, the force balance is always maintained which can leads to the two possible cases as shown in Figure 4(b), where the one is that only the second card starts to move while the third card does not and the other is that both the third and the forth cards are moved as shown in the lower example of Figure 4(b). We would note that we can not uniquely determine which can actually happens. From these discussions, we can say 


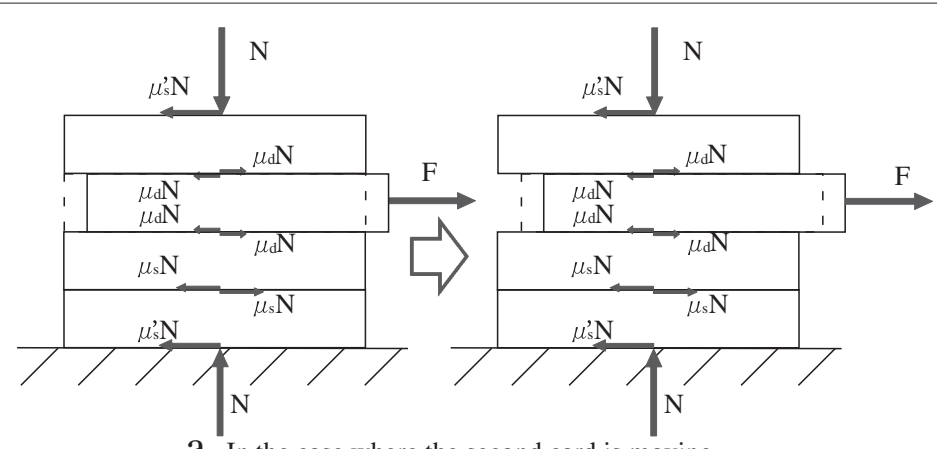

a In the case where the second card is moving

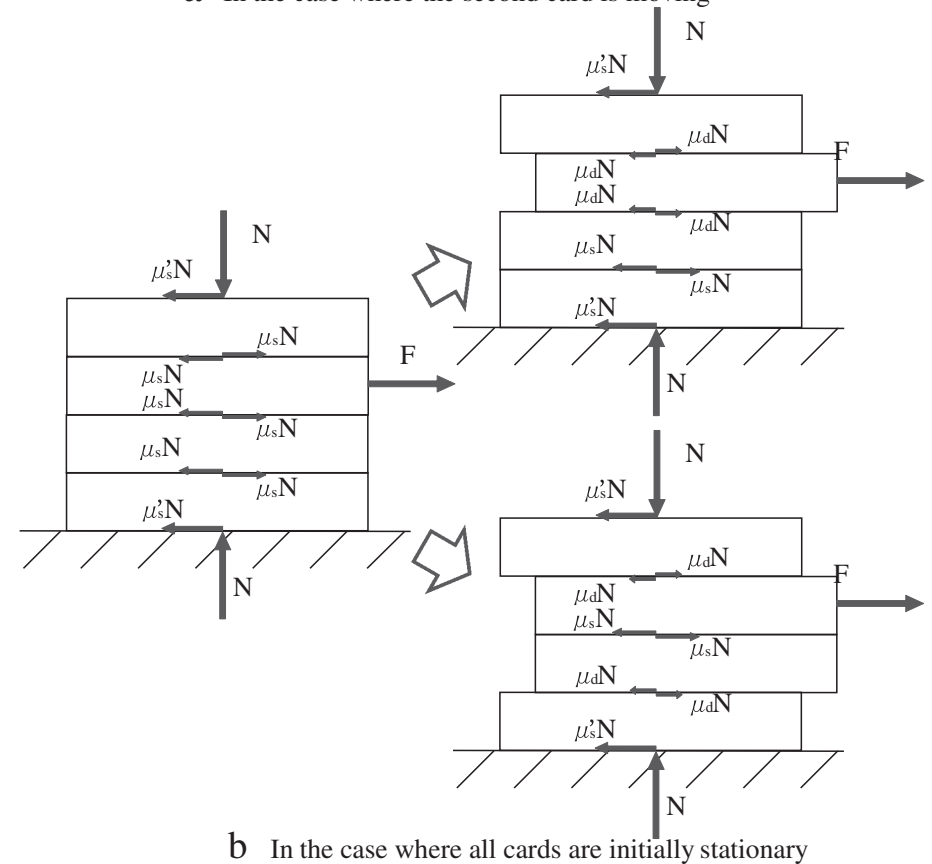

Figure 4 The model of the second-card-dealing.

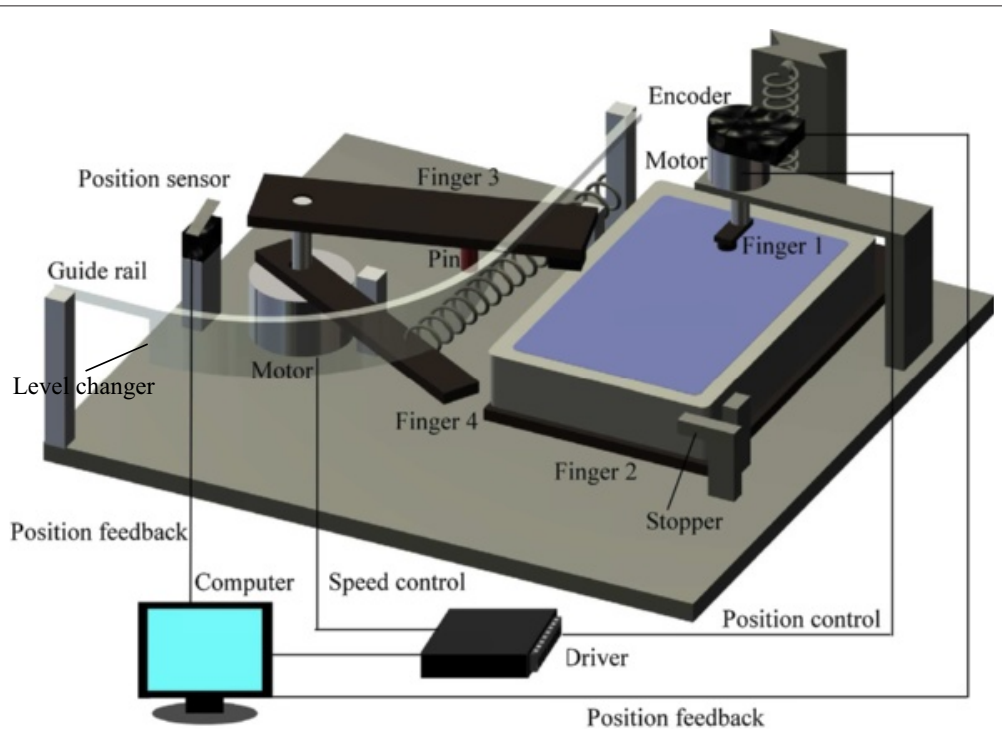

Figure 5 Overview of the experimental system. 
that we can remove the second card without keeping both the top and the third cards stationary if the second card is already in moving initially. However, if this is not the case, it is not guaranteed that we can remove the second card without keeping both the top and the third cards stationary. In summary, in either case, we can keep the top card stationary by increasing $\mu_{s}^{\prime}$, which can be easily achieved
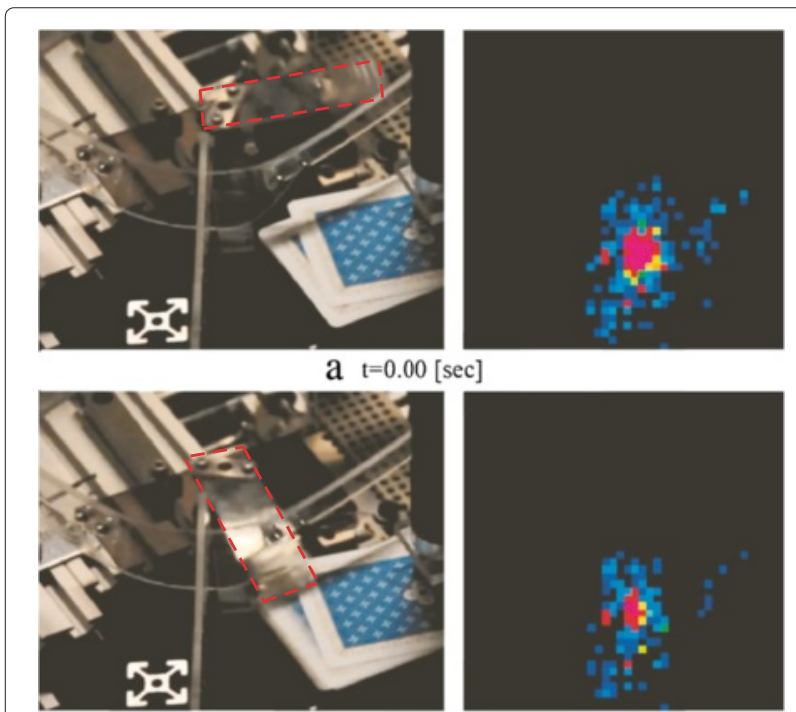

b $\mathrm{t}=0.08[\mathrm{sec}]$

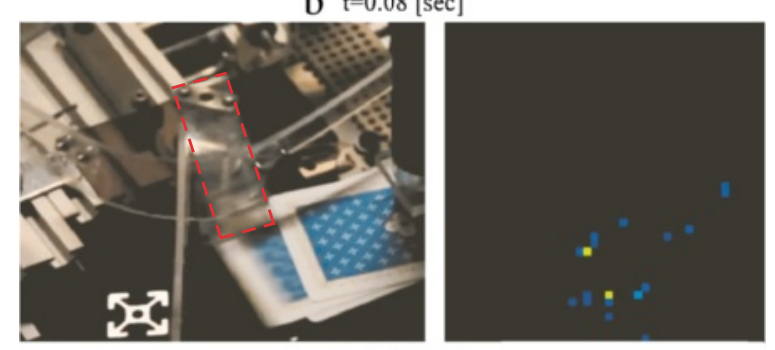

C $\mathrm{t}=0.10[\mathrm{sec}]$

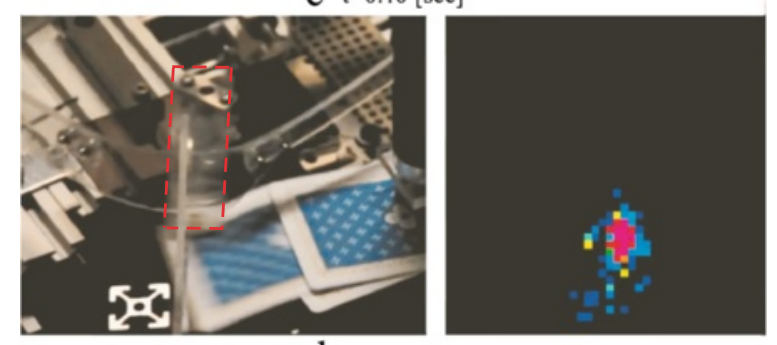

$\mathrm{d} \mathrm{t}=0.12[\mathrm{sec}]$

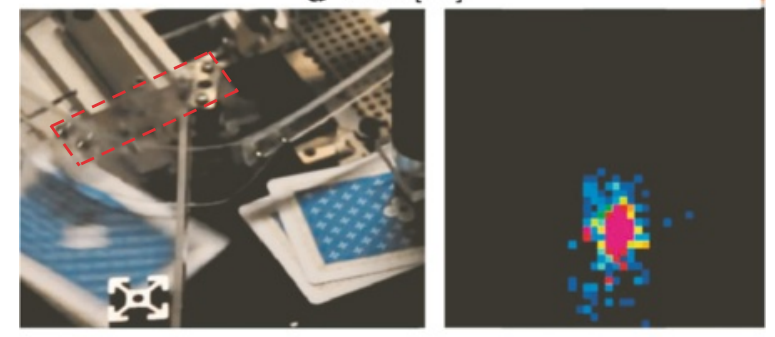

e $t=0.20$ [sec]

Figure 6 Pressure distribution during the second-card-dealing. by covering a soft material around the finger tip. However, it is not guaranteed that we can keep the third card stationary. This result suggests that in designing a robot, we have to consider an appropriate mechanism, so that we can keep the third card stationary.

\section{Methods}

\section{Experimental system}

Figure 5 shows an overview of the developed hyper magician with two active degrees of freedom, where one is for rotating the top card around the axis of Finger 1 perpendicular to the surface of the card and the other is for rotating the Finger 3 continuously. In order to keep all cards except the second card, we implement a stopper as shown in Figure 5. An elastic link is utilized for the Finger 3, so that it can be easily bend along the fixed guide link. With this elastic motion, the Finger 3 can increase the normal force at the contact point between the finger and the second card, which contributes to generating a large pulling force for the second card. By the pin as shown in Figure 5, Finger 4 is moved together with Finger 3, by which two fingers can grab the second card, as shown in Figure 3. A rotational spring is implemented into Finger 4. There is a level changer in the fixed guide link, by which Finger 3 is moved back to its initial finger shape due to its elasticity effect, and at the same time, Finger 4 is released from the pin. Therefore,

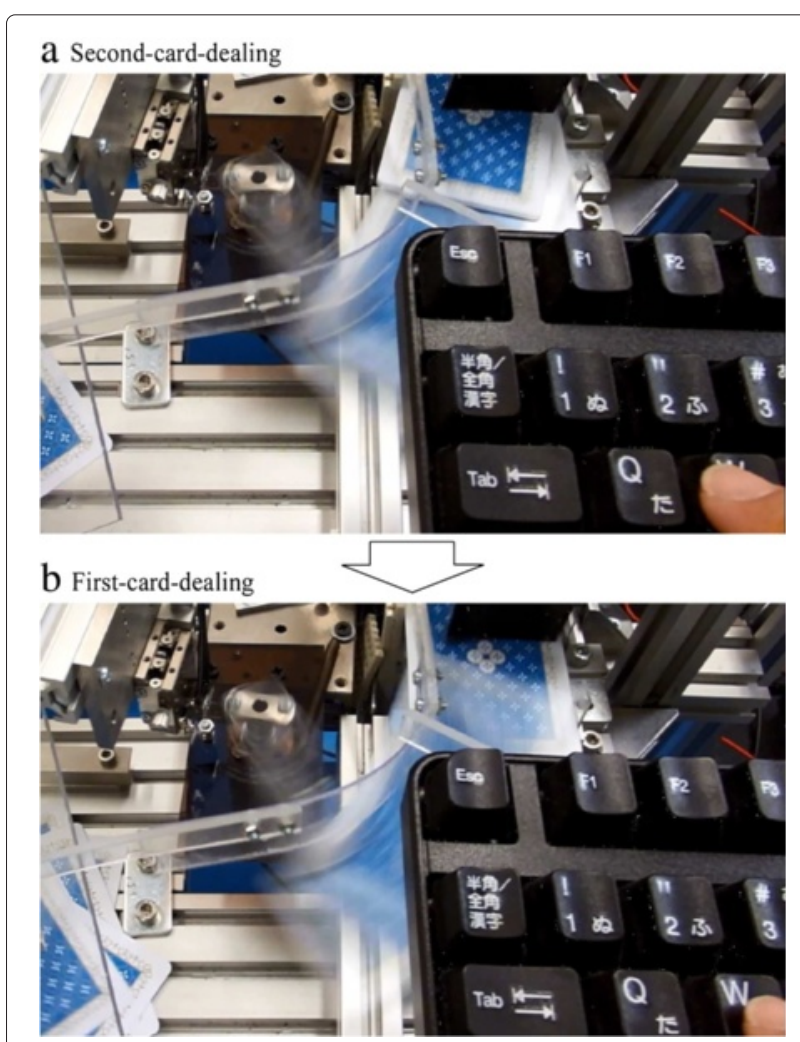

Figure 7 The example of the magic. 
when both Finger 4 and Finger 3 rotate to a prescribed angle, Finger 4 is released from mechanical constraint and automatically moves back to the stand-by position which corresponds to the location of the edge of card block.

\section{Measurement of the pressure distribution between cards}

To examine the pressure distribution between cards during the second-card-dealing, we implement a distributed type pressure sensor between the third and the forth card, just below the contact area of the Finger 1, where the sensing area and the resolution of the pressure sensor are $44 \times 44 \mathrm{~mm}^{2}$ and $1 \mathrm{~mm}$, respectively. The experiment is executed under the card removing velocity of $0.4 \mathrm{~s} / \mathrm{card}$, which corresponds to $38 \%$ of the top speed. Figure 6 shows the experimental results where the right and the left column are pressure distribution and a snap shot obtained by a high speed camera. Figure 6(a) is corresponding to just before Finger 3 touches with the second card. Figure 6(b), (c) and (d) are corresponding to the phase where Finger 3 touches with the second card and makes it slide between the top and the third cards. Figure 6(e) is corresponding to the phase where Finger 3 complete to move the second card. As for the pressure, the sensor output covers relatively large area and shows a high peak due to the grasping force by both Finger 1 and Finger 2. When Finger 3 makes contact with the second card, due to the pushing force by Finger 3, the grasping force by both Finger 1 and Finger 2 decreases. Because of this, pressure becomes weak, as shown in Figure 6(c). The pressure recovers quickly as the Finger 3 complete to remove the second card from the card bundle. We can regard that the pressure distribution is the distribution of normal force. Accordingly, this pressure distribution can be regarded as local friction distribution. Considering this characteristics, we can understand that when the second card moves away from the grasping point by both Finger 1 and Finger 2 , the friction force acted on the second card is drastically reduced. This means that the second card can be easily removed, once it moves away from the grasping point. On the other hand, a large friction force may occur until the second card moves away from the grasping point, which may bring the sliding motion for the third card as easily expected. The stopper can completely avoid such a sliding motion of the third card in the developed robot system.

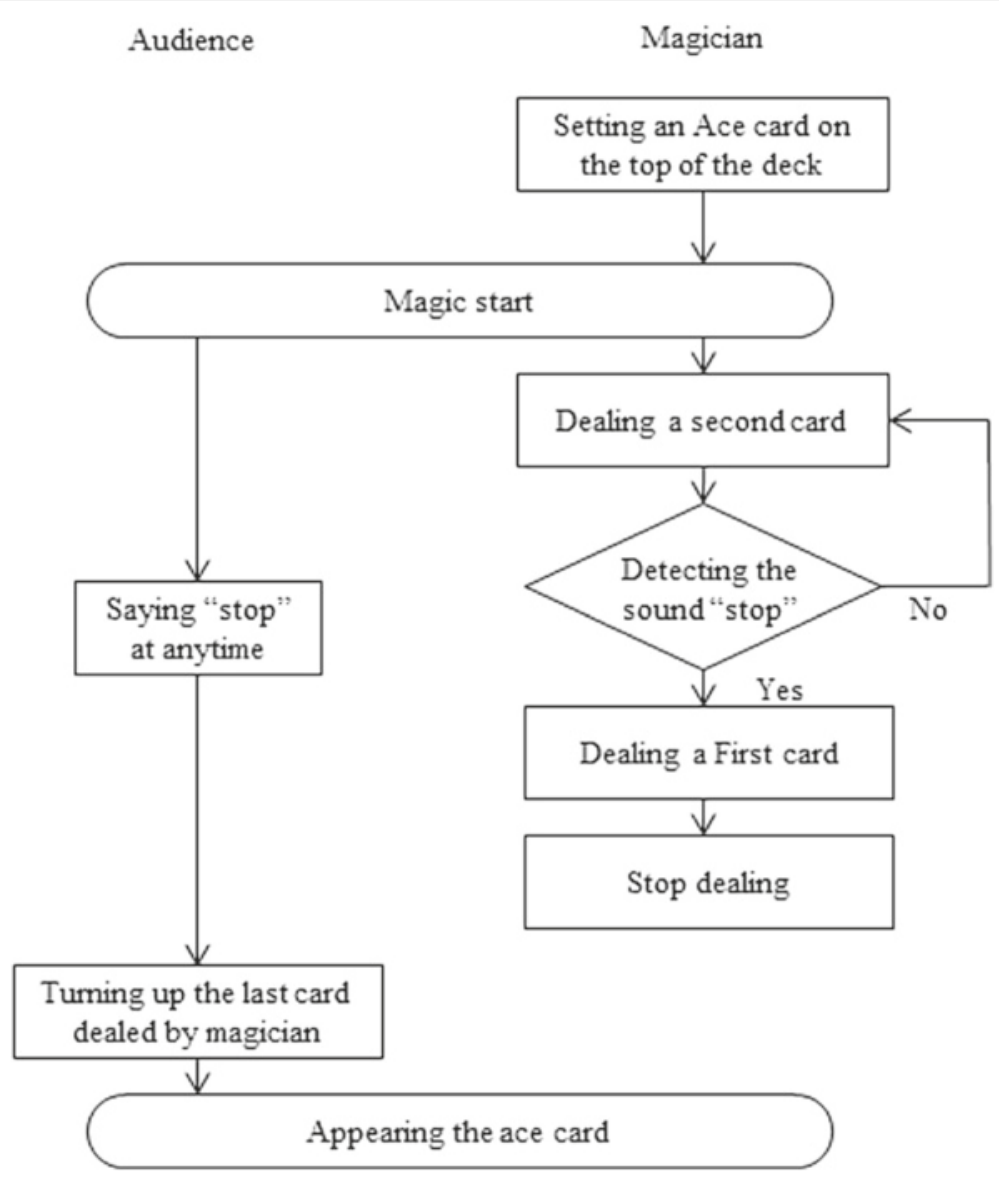

Figure 8 Time chart for the first-card-dealing and the second-card-dealing. 


\section{Results}

\section{Two examples of magic}

The first magic is demonstrated by video (see the Additional file 1: video clip), where the "Ace" is put on the top of the bundle of cards. Then, the robot starts the second-card-dealing continuously and stops the dealing when the predetermined number of cards are dealt. Then, the last card is opened by an audience. The magic is that the last card is always the "Ace". The second magic is demonstrated by video (see the Additional file 1: video clip), where an audience pushes the key board for starting the second card dealing. At any time, he (or she) can push the button for stopping. When the stop signal is detected by the robot, it changes from the second-card-dealing to the first-card-dealing quickly. As a result, the last card is always the one ("Ace") put on the top initially. Two cut scenes are shown in Figure 7 where Figure 7 (a) and (b) are the second-card-dealing after the start button and the first-card-dealing after the stop button.

\section{Trick}

Figure 8 shows two time charts for the first-card-dealing and the second-card-dealing by the robot, respectively. The difference between them is the rotational angle of the top card driven by the Finger 1 . The trick motion whether the robot takes the top card or the second card depends on the selection of time chart of Finger 1 . The time difference of them is $0.04[s]$, which can not be followed by human. We would also note that the processing time with max speed for taking one card from the bundle of cards is only $0.13[s]$ which corresponds to six cards/sec, and it is even faster than human, which also makes human eye difficult to follow.

\section{Discussion}

Figure 9 explains a time chart showing the behavior of both a card magician and an audience enjoying the card magic where an audience can say "stop" at any time while the magician is dealing card one by one. The magic is in such a way that the next card immediately after saying "stop" is always "Ace". The trick is same as the one explained in the second example as shown in Figure 8. Actually, as far as this card magic is concerned, a robot magician can play with more advantage than human.

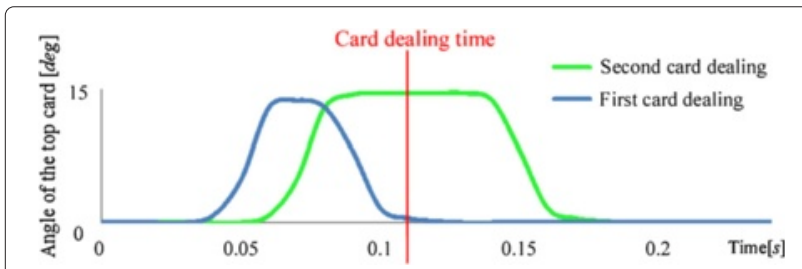

Figure 9 Time chart of the magic.

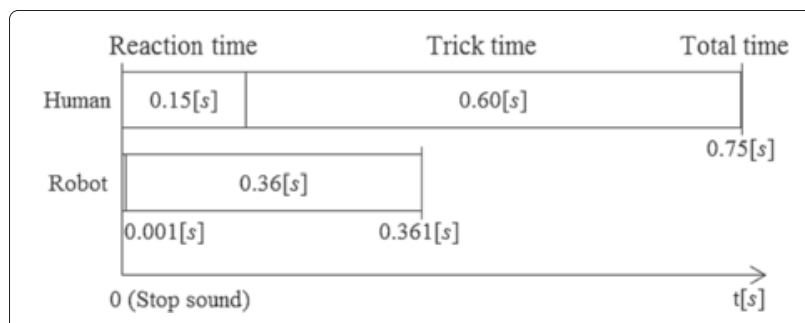

Figure 10 Action time between human and the robot.

Figure 10 shows the time map taken for each motion. For example, it takes only $0.001[s]$ for a robot to start action after hearing a sound saying "stop", while it takes roughly $0.15[s]$ for human [26] to activate his (or her) muscle after hearing "stop", where $0.001[s]$ corresponds to sampling time for robot control. On the other hand, it takes $0.36[s]$ for a robot magician to deal a card, while it takes about $0.6[s]$ for human magician [1]. Totally, a robot magician completes the trick with $0.36[s]$, while human magician do with $0.75[s]$, which is two times more than the robot. Since it is said that the dynamic recognition speed of human eye is less than 0.4[ $s]$ [27], we can say that the robot magician is more advantage than human magician.

Now, let us discuss the technology map for card magic as shown in Figure 11. For discussing such a technology map, there are two important key words where one is "finger dexterity" and the other is "manipulation speed". By using these two key words, we can provide a technology map of card magic where the blue area denote the one where a card magic is successful. As for dexterity, human finger manipulation is so dexterous and skillful, while it is very difficult for even the most advanced robot to open a card just like a human. On the other hand, as for speed,

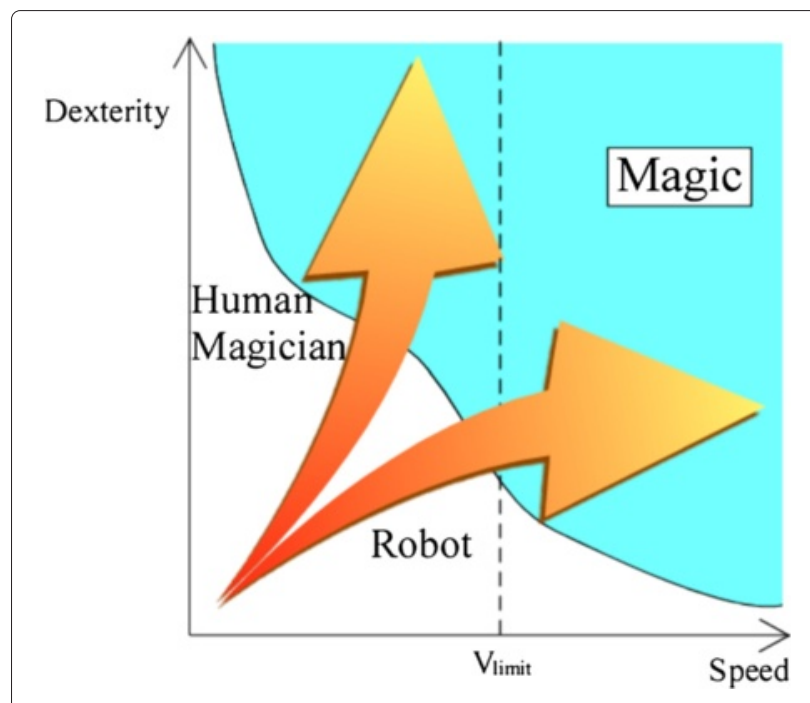

Figure 11 Technology map. 
human have a limitation of finger manipulation speed $V_{\text {limit }}$, while a robot can easily exceed $V_{\text {limit }}$ by implementing a high speed actuator, as shown in Figure 11. Simply speaking, while human magician can complete a trick by skillful finger manipulations within a limitation of $V_{\text {limit }}$, a robot magician can easily complete a trick by speed rather than skillful manipulation. By considering those facts, a possible style of a robot magician is the interactive magic between a robot magician and audience, where the robot magician plays card magic with a high speed, and opening a card is asked to the audience with a higher dexterous manipulation skill. We believe that this type of interactive magic may provide with another card magic entertainment in the future.

\section{Conclusions}

Observing human finger motion during the second-carddealing, we first extract the active degrees of freedom which are definitely necessary for the robot that can achieve a card magic. Focusing on the first and the second card dealing, we discussed the condition where the second card is removed without keeping both the top and the third cards stationary, and showed that it is hard to keep the third card stationary while we can easily achieve to keep the top card stationary. Based on these discussions, we designed and developed the four-fingered robot where two of four fingers have actuators and the remaining fingers do not have. The measurement of pressure distribution during the second-card-dealing showed that the friction force heavily changes during the card manipulation and the mechanical stopper is definitely needed for keeping the second card stationary. As an example, we showed that the robot can achieve a magic by quickly switching the first-card-dealing and the secondcard-dealing.

\section{Additional file}

Additional file 1: In this video, you can see the robot magician capable of taking out the first or second card from the top of a bundle of cards. The robot can achieve card magic with the speed of six cards/sec, which is even faster than human magician.

\section{Competing interests}

The authors declare that they have no competing interests.

\section{Authors' contributions}

RK carried out the main part of the studies and participated in the design of the study. MH carried out the part of the study, the idea of pressure sensing MK participated in its coordination. All authors read and approved the final manuscript.

\section{Acknowledgements}

This work is partially supported by The Ministry of Education, Culture, Sports, Science and Technology (MEXT) of Japan Grant-in-Aid for Challenging Exploratory Research $\sharp 26630098$.
Received: 30 September 2014 Accepted: 8 December 2014

Published online: 11 February 2015

\section{References}

1. (2006) One Handed Bottom Deal - Card Tricks. http://www.youtube.com/ watch?v=PxCNhQBSrIA

2. (2006) Second Deal - Card Tricks. http://www.youtube.com/watch?v= GKY6fhCMcsl

3. (2007) Buckeye (tutorial). http://www.youtube.com/watch?v= vTsr_TrahwM

4. Tamariz J (1987) Theory of False Solutions and The Magic Way. Editorial Frakson Magic Books, Madrid

5. Ganson L (1959) Dai Vernon's Inner Secrets of Card Magic. The Supreme Magic Company, Devon

6. Marlo E (1959) Seconds, Centers, Bottoms, Revolutionary Card Technique Chapter 8, 9, 10. Magic, Inc, Chicago

7. Nuñez D, Tempest M, Viola E, Breazeal C (2014) An Initial Discussion of Timing Considerations Raised During Development of a Magician-Robot Interaction, Timing in Human-Robot Interaction. In: Proceedings of HRI2014 Workshop. ACM/IEEE, Bielefeld

8. Tamura Y, Yano S, Osumi H (2014) Modeling of human attention based on analysis of magic. In: Proceedings of HRI2014. ACM/IEEE, Bielefeld. pp 302-303

9. Howe R, Kao I, Cutkosky M (1988) Sliding of robot fingers under combined torsion and shear loading. In: Proceedings of Robotics and Automation, IEEE, Philadelphia. pp 103-105

10. Kao I, Cutkosky M (1992) Dexterous manipulation with compliance and sliding. Int J Robot Res 11(1):20-40

11. Howe R, Cutkosky M (1996) Practical force-motion models for sliding manipulation. Int J Robot Res 15(6):555-572

12. Goyal S, Ruina A, Papadopoulos J (1991) Planar Sliding with Dry Friction: Part 2. Dynamics of Motion, Wear, No.143, pp 331-352

13. Harada K, Nishiyama J, Murakami Y, Kaneko M (2002) Pushing Multiple Objects Using Equivalent Friction Center, Proc. of IEEE Int.

14. Lynch K, Mason M (1996) Stable pushing: mechanics, controllability, and planning. Int J Robot Res 15(6):533-556

15. Mason M (1986) Mechanics and planning of manipulator pushing operations. Int J Robot Res 5(3):53-71

16. Harada K, Kaneko M, Tsuji T (2000) Rolling based manipulation for multiple objects. In: Proceedings of Robotics and Automation, IEEE, San Francisco. pp 3888-3895

17. Cole A, Hauser J, Sastry S (1989) Kinematics and control of multifingered hands with rolling contact. IEEE Trans. Automatic Control 34(4):398-404

18. Xydas N, Kao I (1999) Modeling and contact mechanics for soft fingers in grasping and manipulation. Int J Robot Res 19(9):941-950

19. Kao I, Yang F (2004) Stiffness and contact mechanics for soft fingers in grasping and manipulation. IEEE Trans Robot Automation 20(1):132-135

20. Salisbury K, Roth B (1983) Kinematics and force analysis of articulated mechanical hands. J Mechan Trans Actuat Des 105(1):35-41

21. Kaneko M, Tanie K (1988) Basic Considerations on the Development of a Multi-fingered Robot Hand with the Capability of Compliance Control. In: Proceedings of Advances in Robot Kinematics, Ljubljana

22. Paetsch W, Kaneko M (1990) A three fingered, multijointed gripper for experimental use. IEEE Int. Workshop on Intell Robot Syst:853-858

23. Jacobsen S, Iversen E, Knutti D, Lohnsan R, Biggers K (1986) Design of the Utah/MIT Dexterous Hand. In: Proceedings of Robotics and Automation, IEEE, San Francisco. pp 2485-2491

24. Kaneko M, Higashimori M, Takenaka R, Namiki A, Ishikawa M (2003) The $100 \mathrm{G}$ capturing robot-too fast to see. IEEE/ASME Trans. Mechatronics 8(1):37-44

25. Higashimori M, Utsumi K, Omoto Y, Kaneko M (2009) Dynamic manipulation inspired by the handling of a pizza Peel. IEEE Trans Robot 25(4):829-838

26. Lipps DB, Galecki AT, Ashton-Miller JA (2011) On the implications of a sex difference in the reaction times of sprinters at the Beijing Olympics. PLoS ONE. DOI: 10.1371/journal.pone.0026141

27. Laming DRJ (1968) Information Theory of Choice-Reaction Times. Academic Press, London 